\title{
MAXIMALITY AND ULTRACOMPLETENESS IN NORMED MODULES ${ }^{1}$
}

ISIDORE FLEISCHER

1. What follows is a drastically stripped down version of a paper completed several years ago. The original intention was to present a careful treatment of the developments centering around the maximality concept in classical valuation theory, using modern ideas and the natural setting of an additive group, and to apply the resulting theory to systematize and simplify some recent investigations into ordered groups. The various referees through whose hands the paper has since passed have finally succeeded in convincing the writer that there is insufficient interest in such a project to justify publication. Accordingly the present version confines itself strictly to an account of the new results obtained.

Even before the first submission of this paper, there had appeared an article by Conrad [C] in which ordered groups are handled from the valuation standpoint, but which does not specifically make use of techniques from valuation theory. Subsequently, while this paper was bottled up in the refereeing process, Gravett [G] rederived Conrad's results, at least for the case of interest here, by generalizing the appropriate valuation theoretic arguments. What is still new in the present paper is the example of a nonunique maximal immediate extension, and the study of normed spaces over fields with a valuation, which can be used to treat ordered spaces over ordered fields.

2. Let $M$ be a module with domain of operators $R$ and $\Lambda$ a linearly ordered set with smallest element 0 . A norm on $M$ is a mapping $V: M \rightarrow \Lambda$, satisfying, for $x, y \in M, \alpha \in R$ :

$$
\begin{aligned}
V(x) & =0 \leftrightarrow x=0, \\
V(x \pm y) & \leqq M a x V(x), V(y), \\
V(\alpha x) & \leqq V(x) .
\end{aligned}
$$

The norm may also be described by the submodules

$$
M_{\lambda}=\{x: V(x) \leqq \lambda\}
$$

which are linearly ordered by inclusion; every element of $M$ is contained in a smallest $M_{\lambda}$.

$M$ is ultracomplete if it is complete in every (not necessarily sepa-

Presented to the Society April 5, 1957 ; received by the editors July 23, 1957.

${ }^{1}$ Partly done under a National Research Council Fellowship. 
rated) topology which has a base at zero of $M_{\lambda}$. The following theorem was proved in the original version of this paper:

THEOREM 1. The following are each necessary and sufficient that $M$ be ultracomplete:

(i) Every family of cosets of $M_{\lambda}$ with the finite intersection property has a nonvoid intersection.

(ii) Every pseudo-convergent sequence has a pseudo-limit.

(iii) The natural mapping of $M$ into any projective limit of $M / M_{\lambda}$ is onto.

(iv) Every quotient module by an intersection of $M_{\lambda}$ is complete in the topology defined by the images of these $M_{\lambda}$.

Here a sequence $\left\{x_{\lambda}\right\}$ (indexed by a subset of $\Lambda$ ) may be defined to be pseudo-convergent if $V\left(x_{\mu}-x_{\lambda}\right) \leqq \lambda$ for $\mu<\lambda$, and to have $x$ as pseudo-limit if $V\left(x-x_{\lambda}\right) \leqq \lambda$; or the definition may be altered by substituting equalities for the inequalities.

For $\lambda \neq 0$, the quotient module of $M_{\lambda}$ by $\bigcup_{\mu<\lambda} M_{\mu}$ is the $\lambda$ th residue module $\bar{M}_{\lambda}$. If $N$ is a submodule of $M, V$ induces a norm on $N$. According to the isomorphism theorem, $\bar{N}_{\lambda}$ may be regarded as a submodule of $\bar{M}_{\lambda}$ : If $\bar{N}_{\lambda}=\bar{M}_{\lambda}$ for all $\lambda, M$ is said to be an immediate extension of $N$. A module admitting no proper immediate extension is maximal. The existence of at least one immediate maximal extension may be shown by transfinite induction.

3. Let $N \subset M$ and $x \in N$. If $\{V(x-y): y \in N\} \subset \Lambda$ has a smallest element, say $V\left(x-y_{0}\right)=\lambda_{0}$, then the $\lambda_{0}$ th residue of $x-y_{0}$ is disjoint from $\bar{N}_{\lambda_{0}}$; if it has no smallest element, there exists a sequence $y_{\lambda} \in N$ such that $V\left(x-y_{\lambda}\right)$ is cofinal to the left. The triangle inequality shows that $y_{\lambda}$ are pseudo-convergent with pseudo-limit $x$; the cofinality shows that $y_{\lambda}$ have no pseudo-limit in $N$.

Theorem 2. Let $N$ be a submodule of $M, x$ an element not in $N$. Then either in the submodule spanned by $x$ and $N$ some element has a residue differing from the corresponding residue of any element of $N$, or $x$ is the pseudo-limit of a pseudo-convergent sequence of $N$ having no pseudolimit in $N$.

Corollary 1. $M$ is an immediate extension of $N$ if and only if every element of $M$ not in $N$ is the pseudo-limit of a pseudo-convergent sequence in $N$ having no pseudo-limit in $N$.

Corollary 2. An ultracomplete module is maximal.

Our proof of the converse of Corollary 2 will be based on the follow- 
ing known lemma valid for modules over a principal ideal ring: ${ }^{2}$

Let $\phi$ be a homomorphism of $M$ in to $\bar{M}$ with kernel $M_{0}$. Then there exists a module $\hat{M}$ containing $M$ and a homomorphism $\widehat{\phi}$ extending $\phi$ which is onto $\bar{M}$ and has kernel $M_{0}$.

To apply the lemma, let $M$ be normed, $M_{0}$ a proper intersection of $M_{\lambda}$ and norm $M / M_{0}$ with $M_{\lambda} / M_{0}{ }^{3}{ }^{3}$ The topological completion $\bar{M}$ of $M / M_{0}$ for this norm becomes an immediate extension if we norm by the closures of $M_{\lambda} / M_{0}$. Using the lemma we now obtain a module $\hat{M}$ which we norm as follows: If $M_{\lambda}$ originally contained $M_{0}$ properly; i.e., if $\lambda$ is a norm associated with $\bar{M}$, we take for $\hat{M}_{\lambda}$ the inverse image under $\hat{\phi}$ of the corresponding module in $\bar{M}$. In the contrary case, $M_{\lambda} \subseteq M_{0}$ and we set $\hat{M}_{\lambda}=M_{\lambda}$. We verify in straightforward fashion that $\hat{M}$ is an immediate extension of $M$. Furthermore, since $\widehat{\phi}$ is onto $\bar{M}, \hat{M}$ properly contains $M$ if $M / M_{0}$ is not complete. Therefore:

Theorem 3. A maximal module over a principal ideal ring is ultracomplete.

CoRollary. Every module over a principal ideal ring has an immediate ultracomplete extension.

4. That an immediate maximal extension need not be unique is shown by the following example.

Let $M$ be the direct sum of countably many copies of $Z_{2}$ (generators $x_{i}$ indexed by the positive integers) and a copy of $Z_{4}$ (generator $x$ ). Set $M_{0}=M ; M_{n}$ is to be the subgroup generated by the $x-x_{k}$ for $k \geqq n>0 ; M_{\omega}$, the subgroup generated by $2 x$, is the intersection of the $M_{n} ; M_{\omega+1}=0$. Since the $M_{\lambda}$ are closed under intersection, there exists a smallest one containing any element, and we have a norm.

The subgroup $N$ of $M$ is to be generated by the $x_{i}$ and $2 x$. Since every element of $M_{n}$ can be converted, by addition of an element of $M_{n+1}$, into an element of $N_{n}=M_{n} \cap N, M$ is an immediate extension of $N$. But $N$ is a vector space over the field of two elements and consequently has an immediate maximal extension which is also such a vector space. The latter cannot be isomorphic to an immediate maximal extension of $M$, which contains an element of order four.

5. The uniqueness of the maximal completion can be established if $R$ is a division ring or in the following more general situation which is not subsumed under our previous formalism.

${ }^{2}$ Eilenberg-MacLane, Ann. of Math. vol. 43 (1942) p. 794, Lemma 23.1.

3 The norm may be obtained from $\Lambda$ by identifying with 0 the initial segment of $\Lambda$ corresponding to $M_{0}$. 
Let $M$ be a vector space over a field $K$ and assume that besides the norm $V$ of $M$ into $\Lambda$ there is also defined a valuation $v$ of $K$ into a linearly ordered multiplicative group augmented by a smallest element $0, \Gamma$, which acts on $\Lambda$ as order-preserving operators: for the product $\gamma \lambda, \gamma \in \Gamma, \lambda \in \Lambda$, we thus postulate:

$$
\begin{aligned}
(\gamma \delta) \lambda & =\gamma(\delta \lambda), \\
\gamma & \leqq \delta \rightarrow \gamma \lambda \leqq \delta \lambda, \\
\lambda & \leqq \mu \rightarrow \gamma \lambda \leqq \gamma \mu .
\end{aligned}
$$

The previous axiom $V(\alpha x) \leqq V(x)$ is now to be replaced by

$$
V(\alpha x)=r^{\prime}(\alpha) V(x) .
$$

In these circumstances we shall say that $M$ is a normed space over $K$.

All that has been proved for normed modules remains valid for normed spaces: only the ultracompleteness of maximal extensions merits special verification. To prove this, let $\left\{x_{\lambda}\right\}$ be a pseudo-convergent sequence without a pseudo-limit in $M$. We shall norm the module $M \oplus K x^{*}$ in such a way that it becomes an immediate extension of $M$, and that $x^{*}$ is a pseudo-limit of the sequence $\left\{x_{\lambda}\right\}$. If $V\left(x_{\lambda}\right) \leqq \lambda$ were true for all $\lambda$, zero would be a pseudo-limit of $\left\{x_{\lambda}\right\}$; therefore for some $\lambda^{\prime}$ we have $V\left(x_{\lambda^{\prime}}\right)>\lambda^{\prime}$. In conjunction with the triangle inequality this yields the constancy of $V\left(x_{\lambda}\right)$ for $\lambda<\lambda^{\prime}$; and this constant value must obviously be $V\left(x^{*}\right) .{ }^{4}$ More generally, given any $k \neq 0$ in $K$ and any $x \in M$ one shows that $V\left(k x_{\lambda}+x\right)$ is eventually constant, and assigns this constant as the norm of $k x^{*}+x$. We omit the straightforward verification that these definitions make $M \oplus K x^{*}$ into a normed space having the required properties.

The following theorem is only a minor extension of Ingleton's [I].

THEOREM 4. Let $M$ and $M^{*}$ be two normed spaces over $K$ with respective norm sets $\Lambda$ and $\Lambda^{*}$; let $\phi$ be an order preserving function from $\Lambda$ into $\Lambda^{*}$ respecting the action of $\Gamma$; and let $f$ be a homomorphism defined on a subspace of $M$ into $M^{*}$ which is dominated by $\phi: V^{*}(f(x)) \leqq \phi(V(x))$. Suppose finally that $M^{*}$ is maximal. Then $f$ has an extension to all of $M$ which is still dominated by $\phi .^{5}$

Proof. By transfinite induction it suffices to extend $f$ one step. Suppose then that $x$ does not belong to the subspace $N$ which is the

${ }^{4}$ Note that for sequences not having zero as a pseudo-limit the sequence determines the norm of the limit.

5 That conversely this property implies ultracompleteness follows from the construction just given. 
domain of $f$. If the residue of $x$ is distinct from the residue of any element of $N$, then $V(x+y) \geqq V(y)$ for every $y \in N$ and we may simply set $f(x)=0$. (Scalar multiples never cause trouble.) If this is true for no element of the space spanned by $N$ and $x$, then $x$ is the pseudo-limit of a pseudo-convergent sequence $y_{\lambda}$ in $N$, not having a pseudo-limit in $N$. The $f\left(y_{\lambda}\right)$ are pseudo-convergent in $M^{*}$ with respect to $\phi(\lambda)$ : since $M^{*}$ is maximal they have a pseudo-limit which we take for $f(x)$. Since for every $y$ there exists $\lambda$ such that $V(x-y)$ $=V\left(y_{\lambda}-y\right)$, this extension has the required property.

Corollary. If $M^{\prime}$ is an immediate extension of $M$ and $M^{*}$ a maximal immediate extension, then the identity on $M$ can be extended to a norm preserving isomorphism of $M^{\prime}$ into $M^{*}$. In particular, any two maximal immediate extensions of $M$ are norm isomorphic.

Proof. Of course, we take for $\phi$ in the theorem the identity on $\Lambda$ and for $f$ the identity on $M$. It remains to show that at each step the extension preserves norms. Now since $M^{\prime}$ and $M^{*}$ are both immediate extensions of $N$ and $f(N)$ we are always dealing with pseudo-convergent sequences not having pseudo-limits in $M$; we have seen that for these the norm of a limit is determined by the sequence, ${ }^{4}$ hence must be the same for $x$ and $f(x)$.

It should be remarked that the image of $M^{\prime}$ in $M^{*}$ is by no means unique; or, another way of putting it, that there exist automorphisms of $M^{*}$ which induce the identity on $M$.

6. We propose, finally, to exhibit the structure of normed spaces over fields with a valuation.

We shall say that subspaces $M_{\rho}$ of $M$ are independent if for any finite sum $\sum x_{\rho}$ of elements chosen from distinct $M_{\rho}$ there holds $V\left(\sum x_{\rho}\right)$ $=\max _{\rho} V\left(x_{\rho}\right)$. If $M_{\rho}$ take nonzero norms in disjoint portions of $\Lambda$, then they are independent by the triangle inequality.

If $\Lambda=\Gamma$ the residue spaces of $M$ are isomorphic vector spaces over the residue class field $R$ of $K$. As in [F] one then shows that elements of $M$ having the same norm whose residues are $R$-independent generate independent one-dimensional subspaces. On the other hand, elements whose residues form a set of $R$ generators for some residue space generate a subspace of which $M$ is an immediate extension.

Henceforth we shall assume: $\gamma \lambda=\lambda$ only if $\lambda=0, \gamma=0$, or $\gamma=1$. This has the effect of making $\gamma \rightarrow \gamma \lambda$ one-to-one for nonzero $\lambda$. The preceding considerations now yield:

TheOREM 5. Let $\left\{\Lambda_{\rho}\right\}$ be the nonzero domains of transitivity of $\Lambda$ under the nonzero action of $\Gamma$; let $\bar{M}_{\rho}$ be any of the residue spaces associ- 
ated with $\Lambda_{\rho}$. Then elements of $M$ whose residues are bases of all the $\bar{M}_{\rho}$ generate independent one-dimensional subspaces of whose sum $M$ is an immediate extension.

Let $\left\{\Lambda_{\rho}\right\}$ be a collection of subsets of $\Lambda$ pairwise intersecting in $\{0\}$ whose union is $\Lambda$. Let $M_{\rho}$ be normed spaces having $\Lambda_{\rho}$ as norm sets. By the well-ordered sum of the $M_{\rho}$ we shall mean that subspace $M$ of $\mathrm{X}_{\rho} M_{\rho}$ for which $V_{\rho}\left(x_{\rho}\right)$ is inversely well-ordered in $\Lambda . M$ is normed by $V(x)=\max _{\rho} V_{\rho}\left(x_{\rho}\right)$. If all the $M_{\rho}$ are maximal, so is $M$.

THEOREM 6. In the notation of Theorem 5 , let $M_{\rho}$ be the subspace of $M$ generated by elements whose residues are a basis of $\bar{M}_{\rho}$, and let $M_{\rho}^{*}$ be its maximal completion. Then the maximal completion of $M$ is the wellordered sum of the $M_{\rho}^{*}$.

To carry the analysis further, it is necessary to investigate maximally complete spaces taking norms in $\Gamma$. This is particularly easy if $K$ is a subfield of a power series field. For in this case we may regard $M$ as an $R$ space and apply Theorem 6 to find that its maximal completion is the well-ordered sum of its $R$ subspaces $M_{\gamma}, 0 \neq \gamma \in \Gamma$. If we choose, as we may, $M_{\gamma}=t^{\gamma} M_{1}$, we recover the action of $K$ on $M^{*}$. The completion is seen to be a space of power series over $M_{1}$; in multiplying by an element of $K$ the coefficients are allowed to operate on the elements of $M_{1}$ and the powers of $t$ combine as in $K$. We obtain the not so surprising consequence that $M^{*}$ admits $K^{*}$ as operators.

Theorem 7. If $K$ is a power series field then each $M_{\rho}^{*}$ of Theorem 6 consists of all power series with coefficients in some residue space belonging to $\Lambda_{\rho}$.

These results have relevance for linearly ordered spaces over linearly ordered fields. We choose for $\Lambda$ the set of cyclic convex subgroups, linearly ordered by inclusion, and for $V$ the mapping which sends every element on the convex subgroup it generates. That the axioms are satisfied is readily verified. The residue spaces may be ordered in a natural way and turn out to be real spaces over the linearly ordered real residue class field: the order in $M$ may be recovered from $\Lambda$ and the order in these spaces. Since $R$ has characteristic zero, $K$ is a power series field [S]. We may leave the translation of the above results for the case at hand to the reader.

\section{BiBLIOGRAPHY}

C. P. F. Conrad, Embedding theorems for abelian groups with valuations, Amer. J. Math. vol. 75 (1953) pp. 1-29. 
F. I. Fleischer, Sur les espaces normés non-archimédiens, Neder. Akad. Wetensch. vol. 16 (1954) pp. 165-168.

G. K. A. H. Gravett, Valued linear spaces, Quart. J. Math. (Oxford) vol. 24. (1955) pp. 309-315.

I. A. W. Ingleton, The Hahn-Banach theorem for non-archimedian valued fields, Proc. Cambridge Philos. Soc. vol. 48 (1952) pp. 41-45.

S. O. F. G. Schilling, The theory of valuations, Amer. Math. Soc., New York, 1950.

NorthWESTERN UNIVERSITY

\section{ON A PROBLEM OF D. R. HUGHES}

\section{E. G. STRAUS AND G. SZEKERES}

D. R. Hughes (Bull. Amer. Math. Soc. vol. 63 (1957) p. 209) has proposed the following problem:

Let $G$ be a group and $p$ a prime. Define $H_{p}(G)$ to be the [normal] subgroup of $G$ generated by all the elements of $G$ which do not have order $p$. Is the following conjecture true: either $H_{p}(G)=\{1\}$ or $H_{p}(G)=G$ or $\left[G: H_{p}(G)\right]=p$ ?

He remarks that the conjecture is true for $p=2$.

In this note we prove Hughes' conjecture for $p=3$.

We shall use the following notation: if $h, g_{1}, \cdots, g_{n} \in G$ and $a_{1}, \cdots, a_{n}$ are integers then

$$
h^{a_{1} g_{1}+\cdots+a_{n} g_{n}}=g_{1}^{-1} h^{a_{1}} g_{1} g_{2}^{-1} h^{a_{2}} g_{2} \cdots g_{n}^{-1} h^{a_{n}} g_{n} .
$$

Lemma 1. If $h \in H_{p}, x \notin H_{p}$ then

$$
h^{1+x+x^{2}+\cdots+x^{p-1}}=1 .
$$

Proof. Since $x^{p-1} \notin H_{p}$, all elements of $H_{p} x^{p-1}$ have order $p$. In particular

$$
\begin{aligned}
1 & =\left(h x^{p-1}\right)^{p}=h x^{p-1} \cdot h x^{p-1} \cdots h x^{p-1} \\
& =h \cdot x^{-1} h x \cdot x^{-2} h x^{2} \cdots x^{-(p-1)} h x^{p-1}=h^{1+x+\cdots+x^{p-1}} .
\end{aligned}
$$

Lemma 2. If $h \in H_{3}$ and $x H_{3} \neq y H_{3}$ then $h^{x+y}=h^{y+x}$.

Proof. By hypothesis $z=x^{-1} y \notin H_{3}$. Hence by Lemma 1

$$
1=h_{1}^{1+z+z^{2}}=h_{1}^{1+z^{2}+s} \quad h_{1} \in H_{3}
$$

Received by the editors July 5, 1957. 